\author{
Cadernos de \\ ESTUDOS LINGǘlSTICOS - (54.1), Campinas, Jan./Jun. 2012
}

\title{
ANÁLISE ACÚSTICA DA FALA SUAVIZADA: ESTUDO DE CASO EM GAGUEIRA ${ }^{1}$
}

\author{
SANDRA MERLO* \\ PLÍNIO ALMEIDA BARBOSA*
}

\begin{abstract}
RESUMO
A suavização é uma estratégia utilizada no tratamento comportamental da gagueira. A estratégia reduz a frequência da gagueira, mas também modifica a prosódia da fala. O objetivo desta pesquisa é comparar diversos parâmetros acústicos da fala suavizada em relação à fala habitual. Para tanto, foi realizado um estudo de caso. O sujeito da pesquisa foi um homem adulto, falante nativo do português brasileiro, com gagueira desde a infância. Ele leu frases-veículo que continham palavras-chave. Ao todo, foram utilizadas 21 palavras-chave: cada uma iniciando com uma das três plosivas não-vozeadas [p, t, k], seguidas por uma das sete vogais orais do português brasileiro [a, eh, e, i, oh, o, u]. As palavras-chave foram do tipo ['CVCV]. Todas as frases foram lidas cinco vezes com a forma habitual e suavizada de fala. Os resultados indicaram que a frequência da gagueira diminuiu com a fala suavizada $(\mathrm{p}<$ $0,001)$. A fase de oclusão das plosivas, as palavras-chave e as frases apresentaram duração reduzida $(\mathrm{p}<0,001)$, enquanto a fase de soltura das plosivas e o tempo de início do vozeamento apresentaram duração aumentada $(\mathrm{p}<0,001)$ na fala suavizada em relação à fala habitual. Esses achados sugerem que a fala suavizada é um modo hipoarticulado de fala. Também houve diminuição da frequência fundamental das vogais e das palavras-chave $(\mathrm{p}<0,001)$, indicando que a fala suavizada é aplicada em nível glótico. Não houve modificações nos três primeiros formantes das vogais, indicando que a fala suavizada não altera a precisão articulatória. Também houve redução da inclinação espectral das sentenças $(\mathrm{p}<0,001)$, sugerindo que a fala suavizada aumenta a energia sonora em altas frequências. Portanto, a suavização não apenas reduz o número de hesitações gaguejadas, mas também modifica significativamente a prosódia da fala.
\end{abstract}

Palavras-chave: Gagueira; suavização; análise acústica.

\footnotetext{
ABSTRACT

"Smooth speech" is a strategy used in the behavioral treatment of stuttering. It reduces stuttering frequency but it also changes speech prosody. The purpose of this research is to compare several acoustical parameters of smooth speech with regard to habitual speech. A case study design was done. The subject was a male adult, native speaker of Brazilian Portuguese, who stuttered since childhood.

*. UNICAMP/IEL - Departamento de Linguística, Grupo de Estudos de Prosódia da Fala. Campinas (SP), Brasil. e-mail: sgmerlo@gmail.com

**. UNICAMP/IEL - Departamento de Linguística, Grupo de Estudos de Prosódia da Fala. Campinas (SP), Brasil. e:mail: pabarbosa.unicampbr@gmail.com

${ }^{1}$ Agradecemos ao sujeito L., que gentilmente aceitou participar desta pesquisa; às fonoaudiólogas Anelise Junqueira Bohnen e Eliana Maria Nigro Rocha, por comentários em uma versão preliminar deste artigo; ao CNPq, pelo financiamento concedido (processo: 140281/2007-0).
} 
MERLO e BARBOSA - Análise acústica da fala suavizada...

He read carrier sentences with embedded keywords. There were 21 keywords: each one beginning with one of three voiceless stops [p, t, k], followed by one of the seven oral vowels of Brazilian Portuguese [a, eh, e, i, oh, o, u]. The keywords were in the form ['CVCV]. All sentences were read five times with the habitual and the smooth manner of speaking. The results indicated that stuttering frequency decreased with smooth speech $(\mathrm{p}<0,001)$. The occlusion phase of stops, the keywords, and the sentences showed decreased duration $(\mathrm{p}<0,001)$, while the burst of stops and the voice onset time showed increased duration $(\mathrm{p}<0,001)$ in smooth speech with regard to habitual speech. This suggests that smooth speech is a hypoarticulated manner of speaking. There was also decrease in the fundamental frequency of vowels and keywords $(\mathrm{p}<0,001)$, suggesting that smooth speech is also applied at the glottal level. There were no changes in the first three formants of vowels, which mean that smooth speech does not change articulatory precision. There was also decrease in the spectral tilt of sentences $(p<0,001)$, which means that smooth speech increases energy in high frequencies. Therefore smooth speech not only reduces stuttered hesitations, but also significantly modifies speech prosody.

Keywords: Stuttering; smooth speech; acoustic analysis.

\section{INTRODUÇÃO}

A gagueira é um distúrbio da fluência da fala, caracterizada pela presença de hesitações específicas: repetições de palavras monossilábicas ou de partes de palavras, alongamentos fônicos iniciais, bloqueios fônicos (Yaruss, 2004). As hesitações típicas da gagueira ocorreriam não devido a um problema com o fone ou com a sílaba que está sendo gaguejado, mas devido à lentidão para iniciar o próximo fone ou a próxima sílaba da sequência (Alm, 2004, 2005). Desta forma, as hesitações gaguejadas seriam compensações de uma dificuldade com o ritmo da fala.

Além das hesitações, a fala gaguejada apresenta outras diferenças em relação à fala fluente. Uma dessas diferenças diz respeito à força de contração muscular empregada na fala: indivíduos portadores de gagueira, principalmente em sua forma crônica, tendem a articular os fones com excessiva força muscular (Ramig \& Bennett, 1997; Yaruss, 2004). Para esses indivíduos, a estratégia de suavização é especialmente indicada como recurso terapêutico (Prins, 1997). Suavizar significa diminuir o grau de tensão muscular dos articuladores durante a fala, fazendo com que se toquem mais levemente (Andrade, 1999; Meira, 1997; Ramig \& Bennett, 1997).

Saber distinguir os níveis de contração muscular é imprescindível para a aprendizagem e a manutenção da fala suavizada. Portanto, para facilitar a aprendizagem da suavização, utiliza-se, em um primeiro momento, uma outra estratégia denominada prática negativa. A prática negativa consiste em falar com vários graus de tensão muscular (Andrade, 1999; Meira, 1997; Ramig \& Bennett, 1997). Por exemplo, falar uma palavra com muita tensão, depois com tensão moderada e, por último, com tensão mínima. Ao contrário da suavização, esta técnica é de uso restrito ao ambiente clínico. A repetição desta prática auxilia o paciente a perceber e apreender as diferenças cinestésicas entre os diversos graus de tensão muscular.

O treino específico com suavização é iniciado quando o paciente progrediu 
significativamente na propriocepção. No princípio, a suavização é totalmente voluntária, mas, com execução rotineira, torna-se mais automatizada. Inicialmente, o treino é altamente estruturado, partindo do nível lexical, passando pelo nível frasal e chegando ao textual (Andrade, 1999; Meira, 1997; Ramig \& Bennett, 1997). Com o aumento da maestria no uso da estratégia, são elaboradas tarefas explícitas de transferência para ambientes e situações fora da clínica.

A suavização está entre os métodos comportamentais mais eficazes para o tratamento da gagueira (Bothe, Davidow, Bramlett \& Ingham, 2006). Foi testada em diversas faixas etárias (mais especificamente, em sujeitos de 7 a 58 anos de idade) e melhora a fluência da fala tanto no pós-tratamento imediato quanto em longo prazo (Bothe et al., 2006). Além disso, a suavização melhora aspectos sociais e emocionais no pós-tratamento imediato e em longo prazo, mesmo a abordagem não incluindo componentes sociais e emocionais (Bothe et al., 2006).

O uso da suavização provoca mudanças diretas na maneira de falar. Seu principal objetivo é reduzir o número de hesitações gaguejadas (Bothe et al., 2006), mas também ocorrem mudanças em relação às características acústicas da fala. Estudos anteriores sugeriram que a "marca acústica" da suavização seria o alongamento de vogais (Blomgren, Robb \& Chen, 1998; Riley \& Ingham, 2000). Entretanto, outros parâmetros acústicos também parecem ser modificados pelo uso da suavização. Análises de oitiva realizadas pelos autores sugeriram que a fala suavizada soa como mais lenta, mais grave, mais monótona e com alguns segmentos articulados de maneira imprecisa em comparação com a fala habitual.

Assim, o objetivo deste trabalho é examinar características acústicas da fala suavizada em relação à fala habitual. Foi realizado um estudo de caso, analisandose quatro parâmetros fonético-acústicos. O parâmetro "taxa de elocução" foi selecionado, porque a gagueira tende a afetar a duração de unidades do tamanho da sílaba, de fones e também de elementos subsegmentais (como o voice onset time, VOT), sendo o efeito mais pronunciado conforme aumenta a gravidade da gagueira (Arcuri, Osborn, Schiefer \& Chiari, 2009; Colacicco, Botelho, Schiefer \& Osborn, 2005). O parâmetro "frequência fundamental" (F0) foi selecionado, porque adultos com gagueira tendem a apresentar reduções na média ou na variabilidade de F0 em comparação com adultos sem o distúrbio (Bosshardt, Sappok, Knipschild \& Holscher, 1997; Denny \& Smith, 1997) e também para comparar indiretamente o grau de tensão muscular das pregas vocais com e sem o uso da suavização. O parâmetro "frequência de formantes" (F1, F2 e F3) foi selecionado, porque adultos com gagueira podem apresentar reduções nos valores de F2 em relação a adultos sem gagueira (Blomgren et al., 1998); além disso, a inclusão de F1 e F3 tem a finalidade de testar se estes formantes também são afetados pela suavização. Finalmente, o parâmetro "inclinação espectral" foi selecionado para medir a intensidade sonora em altas e baixas frequências, a fim de avaliar se ocorrem reduções significativas da intensidade com o uso da suavização. 
MERLO e BARBOSA - Análise acústica da fala suavizada...

\section{MÉTODOS}

\subsection{Sujeito}

\subsubsection{Perfil do sujeito}

$\mathrm{Na}$ época da coleta de dados, L. tinha 21 anos. É um indivíduo do sexo masculino, brasileiro, falante nativo do português, cursou o ensino médio e apresenta gagueira desde os 10 anos. O paciente procurou tratamento fonoaudiológico espontaneamente, porque julgava que precisava de maior fluência em seu novo emprego.

\subsubsection{Avaliação fonoaudiológica}

A avaliação consistiu na aplicação de protocolo de risco para a gagueira (Andrade, 1999), coleta de amostra espontânea de fala, coleta de amostra de leitura em voz alta, triagem de processamento auditivo central (Pereira, 1993) e avaliação do sistema miofuncional oral (Marchesan, 1998).

A aplicação do protocolo de risco (Andrade, 1999) indicou que L. apresenta histórico familiar para o distúrbio (um irmão mais novo gagueja e o bisavô materno também gaguejava), não apresenta histórico mórbido pré, peri e pósnatal, apresenta fatores psicológicos associados à gagueira (tanto familiares, como individuais) e possui fatores estressantes associados.

A análise da amostra de fala espontânea revelou que $18 \%$ da fala foi preenchida por hesitações: 14\% com hesitações comuns e 4\% com hesitações gaguejadas. A taxa de elocução foi de 240 sílabas por minuto. Concomitantemente à emissão verbal, ocorreram movimentos involuntários (piscar de olhos e tremor mandibular). Na leitura em voz alta, $6 \%$ do texto foi preenchido com hesitações: $1 \%$ com hesitações comuns e 5\% com hesitações gaguejadas. A taxa de elocução foi de 271 sílabas por minuto. Não foram observados movimentos involuntários durante a leitura. De acordo com Andrade (2006), o intervalo de confiança da frequência de rupturas em situações de fala espontânea para sujeitos do sexo masculino entre 18 e 28 anos é de até $13 \%$, sendo até 3\% de hesitações gaguejadas. Quanto à taxa de elocução, a mesma autora refere que o esperado também em situações de fala espontânea para sujeitos do sexo masculino entre 18 e 28 anos está entre 167 e 227 sílabas por minuto ${ }^{2}$.

\subsubsection{Terapia fonoaudiológica}

A terapia para fluência seguiu princípios de promoção da fluência e de diminuição da gagueira (Ramig \& Bennett, 1997). Na época da gravação, L.

2. Os resultados do sujeito estão fora dos intervalos de confiança apresentados por Andrade (2006). Entretanto, não necessariamente o sujeito está fora da faixa de variação esperada. Apenas um teste estatístico, comparando os resultados do sujeito com a distribuição utilizada por Andrade (2006), indicaria se realmente o sujeito apresenta hesitações e taxa de elocução acima do esperado em relação a seus pares. 
Cadernos de Estudos Lingüísticos (54.1) - Jan./Jun. 2012

havia começado a treinar a suavização há dois meses (estratégia de promoção da fluência); também estava sendo utilizada a dessensibilização (estratégia de diminuição da gagueira). No julgamento de oitiva da fonoaudióloga e do próprio paciente, L. conseguia utilizar a suavização durante a leitura em voz alta, deixando sua fala soar naturalmente. Entretanto, apresentava dificuldades na utilização da estratégia durante a fala espontânea, porque não conseguia utilizá-la em alguns momentos em que necessitava e nem sempre a fala soava natural; a percepção de oitiva indicava que cinco fatores eram modificados durante a fala suavizada de L.: a taxa de elocução parecia lentificada, pitch e loudness pareciam reduzidos e havia momentos de imprecisão articulatória. Essas ocorrências motivaram a realização deste estudo.

\subsection{Procedimentos}

Para a gravação do material, L. esteve sentado. Em sua frente, foi posicionado o microfone Philips MD 195, que estava conectado ao micro-system AIWA CADW258. As falas foram gravadas em fita-cassete. Não foi possível realizar a gravação em cabine acústica. A gravação foi feita na sala de terapia, a qual esteve relativamente silenciosa e possuía materiais que propiciavam a refração sonora.

Três listas de dissílabos iniciados por [p], [t] e [k] foram elaboradas. A razão da escolha destes fones foi a maior dificuldade de L. em suavizar palavras iniciadas por plosivas. As palavras-alvo foram inseridas em frases-veículo e continham as sete vogais orais em posição tônica do português brasileiro (PB) (Tabela 1).

Tabela 1: Dissílabos iniciados por plosivas não-vozeadas e inseridos em frases-veículo utilizadas neste estudo.

\begin{tabular}{cccc}
\hline Vogais & Fone $[\mathbf{p}]$ & Fone $[\mathbf{t}]$ & Fone $[\mathbf{k}]$ \\
\hline$[a]$ & Diga PARA baixinho. & Diga TARA baixinho. & Diga CARO baixinho. \\
{$[e]$} & Diga PÊRA baixinho. & Diga TERES baixinho. & Diga QUEIRO baixinho. \\
{$[\varepsilon]$} & Diga PÉRA baixinho. & Diga TERA baixinho. & Diga QUERO baixinho. \\
{$[i]$} & Diga PIRA baixinho. & Diga TIRA baixinho. & Diga QUIRO baixinho. \\
{$[o]$} & Diga PORES baixinho. & Diga TOURO baixinho. & Diga CORO baixinho. \\
{$[0]$} & Diga PORO baixinho. & Diga TORA baixinho. & Diga CORO baixinho. \\
{$[u]$} & Diga PURA baixinho. & Diga TURBA baixinho. & Diga CURO baixinho. \\
\hline
\end{tabular}

As palavras "touro" e "queiro", embora escritas com ditongo na primeira sílaba, não foram pronunciadas como tal: foram pronunciadas apenas com vogal média-alta (['toru] e ['kerv]). Caso contrário, não seria possível comparar os dados destas duas palavras-alvo com as demais.

As frases foram impressas em cartões de $10 \times 15 \mathrm{~cm}$. Os cartões eram apresentados aleatoriamente, tendo sido colhidas cinco amostras de cada frase em cada modo de fala (habitual ou suave), totalizando 210 frases. Em uma primeira sessão, foram colhidas todas as amostras de fala habitual e, em uma segunda sessão, todas as amostras de fala suave. 
MERLO e BARBOSA - Análise acústica da fala suavizada...

A digitalização das frases foi realizada utilizando o software Praat 5.1.12 (Boersma \& Weenink, 2009), com som mono, taxa de amostragem a 22,5 kHz e taxa de quantização de 16 bits.

Em um primeiro momento, foi realizada a quantificação das hesitações gaguejadas do corpus; o objetivo era comparar o número de eventos de gagueira entre fala habitual e suave. Também foi realizada análise de oitiva da fala suavizada em relação à habitual, atentando-se para taxa de elocução, pitch, loudness e precisão articulatória ${ }^{3}$.

Em seguida, foi realizada análise acústica, a qual constituiu-se de:

1. Medida da duração da vogal reduzida [e] da palavra anterior à palavra-alvo ("Diga para baixinho"), delimitada como sendo desde o início até o final do seu padrão formântico.

2. Medida da duração das plosivas não-vozeadas das palavras-alvo ("Diga para baixinho"), delimitada como sendo desde o início da fase de oclusão (silêncio) até o início do padrão formântico da vogal seguinte.

3. Medida da duração da fase de oclusão das plosivas não-vozeadas, delimitada como sendo desde o início do silêncio até o início da explosão.

4. Medida da duração da fase de soltura das plosivas não-vozeadas, delimitada como sendo desde o início da explosão até o início do padrão formântico da vogal seguinte.

5. Medida do VOT das plosivas não-vozeadas das palavras-alvo, delimitado como sendo desde o início da explosão até o início do ciclo glotal da vogal seguinte.

6. Medida da duração das vogais tônicas das palavras-alvo ("Diga para baixinho"), delimitada como sendo desde o início até o final do seu padrão formântico.

7. Medida da duração da vibrante simples das palavras-alvo ("Diga para baixinho"), delimitada como sendo desde o início até o final do seu padrão formântico.

8. Medida da duração das palavras-alvo ("Diga para baixinho"), delimitado a partir do início da fase de oclusão da plosiva não-vozeada até o término do padrão formântico da vogal pós-tônica da palavra-alvo.

9. Medida da duração da frase completa ("Diga para baixinho"), do primeiro ao último fone. Em algumas frases, o [d] de "diga" foi desvozeado; nesses casos, a medição não pôde ser realizada.

10. Captura da frequência fundamental no início e no meio das vogais tônicas das palavras-alvo ("Diga para baixinho").

11. Captura da frequência fundamental ao longo das palavras-alvo ("Diga para baixinho").

12. Captura de F1, F2 e F3 no início e no meio das vogais tônicas das palavras-alvo ("Diga para baixinho").

13. Captura da inclinação espectral ao longo das frases ("Diga para baixinho"). A inclinação espectral foi definida como a diferença de intensidade (em dB) entre altas e baixas frequências. O corte entre as duas faixas de frequências foi estabelecido pelo valor médio do F2 da vogal tônica [a] das ocorrências de "para", "tara" e "caro" realizadas pelo sujeito. A média resultante foi de $1400 \mathrm{~Hz}$. As medidas de inclinação espectral foram obtidas com o uso de script específico no software Praat 5.1.12 (Boersma \& Weenink, 2009).

3. Por "precisão ou imprecisão articulatória", referimo-nos à produção de segmentos com ou sem as características articulatórias esperadas. Por exemplo, quando uma plosiva é articulada sem suas fases características de oclusão e soltura, mas com ruído de fricção, a articulação é considerada imprecisa. 
Os valores de frequência fundamental foram calculados automaticamente pelo software supracitado através do método de autocorrelação, com pitch mínimo de $75 \mathrm{~Hz}$ e pitch máximo de $350 \mathrm{~Hz}$. Os valores dos formantes também foram calculados automaticamente pelo software através do método LPC (linear predictive coding), assumindo-se cinco formantes em $5000 \mathrm{~Hz}$.

\subsection{Análise Estatística}

Foram empregados os testes Kolmogorov-Smirnov, qui-quadrado e de proporções para comparar os dados da fala habitual com a suavizada, assumindose $\mathrm{p}<0,05$.

\section{RESULTADOS}

\subsection{Hesitações Gaguejadas}

Foram identificados $26(8 \%)$ eventos de gagueira com a fala habitual. Não houve hesitações gaguejadas com a fala suavizada. Esta diferença é estatisticamente significativa $\left(\mathrm{p}<10^{-4}\right)$.

\subsection{Análise De Oitiva}

De maneira geral, a fala suavizada soou mais lenta, com pitch mais baixo (mais grave), com menor variação de pitch (mais monótona), com loudness mais baixo (mais fraca) e com alguns segmentos articulados de forma imprecisa quando comparada com a fala habitual.

\subsection{Análise Acústica}

\subsubsection{Duração}

A duração das plosivas não-vozeadas, da fase de oclusão das plosivas, das palavras-alvo e das frases foram significativamente menores na fala suavizada em comparação com a fala habitual. A duração da fase de soltura das plosivas e do VOT foram significativamente maiores na fala suavizada em comparação com a fala habitual. A duração da vogal reduzida [e] da palavra "diga", das vogais tônicas e da vibrante simples não se modificaram da fala habitual para a suavizada (Tabela 2).

Não houve diferença estatisticamente significativa entre a duração da fase de soltura das plosivas não-vozeadas e o VOT $\left(\mathrm{c}^{2}=128, \mathrm{p}=0,99\right)$, indicando sincronia entre a fase de soltura e o início do vozeamento.

$\mathrm{Na}$ fala suavizada, houve cinco ocorrências de plosivas fricativizadas, todas com o fone [p].

Além disso, houve 18 ocorrências na fala habitual e 28 ocorrências na fala suavizada de desvozeamento do [d] de "diga". A diferença não é significativa no 
MERLO e BARBOSA - Análise acústica da fala suavizada...

teste de proporções bicaudal $(\mathrm{p}=0,08)$.

Tabela 2: Dados de duração nas duas situações de fala. n.s. = não-significativo.

\begin{tabular}{|c|c|c|c|c|}
\hline & & $N$ & Média (desvio-padrão) & $p$ \\
\hline \multirow{2}{*}{ Vogal reduzida } & Fala habitual & 87 & $108(17) \mathrm{ms}$ & \multirow{2}{*}{ n.s. } \\
\hline & Fala suavizada & 77 & $110(22) \mathrm{ms}$ & \\
\hline \multirow{2}{*}{ Plosivas não-vozeadas } & Fala habitual & 100 & $280(99) \mathrm{ms}$ & \multirow{2}{*}{$<0,001$} \\
\hline & Fala suavizada & 100 & $197(24) \mathrm{ms}$ & \\
\hline \multirow{2}{*}{ Fase de oclusão } & Fala habitual & 100 & $243(100) \mathrm{ms}$ & \multirow{2}{*}{$<0,001$} \\
\hline & Fala suavizada & 96 & 149 (24) $\mathrm{ms}$ & \\
\hline \multirow{2}{*}{ Fase de soltura } & Fala habitual & 100 & $37(18) \mathrm{ms}$ & \multirow{2}{*}{$<0,001$} \\
\hline & Fala suavizada & 96 & $48(21) \mathrm{ms}$ & \\
\hline \multirow{2}{*}{ VOT } & Fala habitual & 100 & $35(18) \mathrm{ms}$ & \multirow{2}{*}{$<0,001$} \\
\hline & Fala suavizada & 96 & $45(21) \mathrm{ms}$ & \\
\hline \multirow{2}{*}{ Vogais orais tônicas } & Fala habitual & 104 & $185(32) \mathrm{ms}$ & \multirow{2}{*}{ n.s. } \\
\hline & Fala suavizada & 105 & $179(30) \mathrm{ms}$ & \\
\hline \multirow{2}{*}{ Vibrante simples } & Fala habitual & 104 & $33(9) \mathrm{ms}$ & \multirow{2}{*}{ n.s. } \\
\hline & Fala suavizada & 105 & $31(9) \mathrm{ms}$ & \\
\hline \multirow{2}{*}{ Palavra-alvo } & Fala habitual & 87 & $615(122) \mathrm{ms}$ & \multirow{2}{*}{$<0,001$} \\
\hline & Fala suavizada & 77 & $511(39) \mathrm{ms}$ & \\
\hline \multirow{2}{*}{ Frase } & Fala habitual & 87 & $1838(259) \mathrm{ms}$ & \multirow{2}{*}{$<0,001$} \\
\hline & Fala suavizada & 77 & $1600(103) \mathrm{ms}$ & \\
\hline
\end{tabular}

\subsubsection{Frequência fundamental e de formantes}

A frequência fundamental diminuiu significativamente na fala suavizada em comparação com a habitual, tanto em relação ao início e ao meio das vogais tônicas das palavras-alvo, quanto em relação às palavras-alvo como um todo (Tabela 3).

Os formantes F1, F2 e F3 não se modificaram no início e no meio das vogais tônicas das palavras-alvo (Tabela 3).

Tabela 3: Dados de frequência fundamental e de formantes nas duas situações de fala. n.s. = nãosignificativo.

\begin{tabular}{|c|c|c|c|c|c|}
\hline & & & $N$ & Média (desvio-padrão) & $p$ \\
\hline \multirow{4}{*}{ Fo } & \multirow{2}{*}{ Vogais tônicas } & Fala habitual & 187 & $144(12) \mathrm{Hz}$ & \multirow{2}{*}{$<0,001$} \\
\hline & & Fala suavizada & 193 & $127(11) \mathrm{Hz}$ & \\
\hline & \multirow{2}{*}{ Palavras-alvo } & Fala habitual & 2305 & $148(17) \mathrm{Hz}$ & \multirow{2}{*}{$<0,001$} \\
\hline & & Fala suavizada & 2150 & $129(13) \mathrm{Hz}$ & \\
\hline \multirow{2}{*}{ F1 } & \multirow{2}{*}{ Vogais tônicas } & Fala habitual & 210 & $450(133) \mathrm{Hz}$ & \multirow{2}{*}{ n.s. } \\
\hline & & Fala suavizada & 210 & $442(128) \mathrm{Hz}$ & \\
\hline \multirow{2}{*}{$F 2$} & \multirow{2}{*}{ Vogais tônicas } & Fala habitual & 210 & $1537(382) \mathrm{Hz}$ & \multirow{2}{*}{ n.s. } \\
\hline & & Fala suavizada & 210 & $1540(389) \mathrm{Hz}$ & \\
\hline \multirow{2}{*}{$F 3$} & \multirow{2}{*}{ Vogais tônicas } & Fala habitual & 210 & $2481(244) \mathrm{Hz}$ & \multirow{2}{*}{ n.s. } \\
\hline & & Fala suavizada & 210 & $2484(211) \mathrm{Hz}$ & \\
\hline
\end{tabular}

\subsubsection{Inclinação espectral}

A inclinação espectral diminuiu significativamente em módulo na fala 
suavizada em comparação com a habitual. Em relação às frases como um todo, o fone que geralmente apresentava menor inclinação espectral era a fricativa [S] de "baixinho". Em relação às palavras-alvo, a região que geralmente apresentava menor inclinação espectral era a soltura das plosivas não-vozeadas (Tabela 4).

Tabela 4: Dados de inclinação espectral nas duas situações de fala.

\begin{tabular}{cccc}
\hline & $N$ & Média (desvio-padrão) & $p$ \\
\hline Fala habitual & 105 & $-15(3) \mathrm{dB}$ & $<0,001$ \\
Fala suavizada & 105 & $-13(2) \mathrm{dB}$ & \\
\hline
\end{tabular}

\section{DISCUSSÃO}

\subsection{Duração}

As plosivas não-vozeadas da fala suavizada apresentaram duração 30\% menor em comparação com as da fala habitual. Esta diferença deveu-se especificamente pela redução de $39 \%$ na duração da fase de oclusão. Isso pode ser explicado pela menor ocorrência de gagueira na fala suavizada, tendo em vista que os bloqueios incidiram sobre a fase de oclusão, aumentando a duração desta fase. Por outro lado, o aumento de $23 \%$ na duração da fase de soltura das plosivas pode ser explicado pela diminuição das pressões intraoral e subglótica, que fizeram com que a soltura ocorresse mais lentamente (Imbrie, 2005).

Não houve diminuição da duração da vogal reduzida imediatamente anterior às plosivas não-vozeadas. Em PB, a unidade de vogal a vogal (VV) é a menor unidade de planejamento rítmico (Barbosa, 2006). A duração dos fones que compõem uma unidade VV depende da natureza desses fones e da relação entre eles. Por exemplo, quando a unidade VV é composta por uma vogal e uma plosiva, a duração vocálica é maior se a plosiva for vozeada e menor se for não-vozeada (Barbosa, 2006). Desta forma, ocorrem compensações duracionais no interior da unidade VV. Por outro lado, sabe-se que essas compensações não ocorrem quando há foco em uma palavra específica (Barbosa, 2006). Nesses casos, o foco ocasiona aumento de duração apenas dos segmentos que compõem a sílaba tônica da palavra que está sendo focada. Um processo similar pode ter ocorrido no experimento realizado, tendo em vista que a frase-veículo era sempre a mesma e que as palavras-alvo eram os elementos modificados (e, portanto, focados).

Houve aumento de $22 \%$ na duração do VOT da soltura da plosiva nãovozeada até o início da vogal tônica. O F0 influencia a duração do VOT nas plosivas não-vozeadas: quanto menor o F0, maior o VOT (McCrea \& Morris, 2005). Na fala suavizada, o F0 da vogal tônica foi menor em comparação com o da fala habitual, indicando diminuição da tensão glótica e, portanto, sendo necessário tempo adicional para elevar a tensão em grau suficiente para iniciar a vibração da pregas vocais (McCrea \& Morris, 2005).

Também houve sincronia entre a fase de soltura das plosivas não-vozeadas e o VOT. Oliveira (2007) não encontrou diferenças entre o tempo de início da fonação 
MERLO e BARBOSA - Análise acústica da fala suavizada...

e a soltura da plosiva [p] quando comparou as emissões de adultos com e sem gagueira. Assim, não há falta de sincronia entre esses fatores na fala gaguejada.

As vogais tônicas e a vibrante simples das palavras-alvo não apresentaram mudanças de duração da fala habitual para a suavizada. No caso das vogais, a redução da tensão muscular não foi suficiente para ocasionar redução do deslocamento da língua. No caso da vibrante simples, é possível que não tenha havido modificação deste segmento, porque é um fone com duração invariante em PB (Barbosa, 2006).

Também houve redução de $17 \%$ na duração das palavras-alvo e de $13 \%$ na duração das frases na fala suavizada em comparação com a fala habitual.

De maneira geral, os dados de duração são compatíveis com a chamada "fala hipoarticulada" (Farnetani, 1997; Harnsberger et al., 2008), o que é coerente com a instrução fornecida ao sujeito (articular com mais suavidade). Tendo em vista que a duração fônica está diretamente correlacionada com a amplitude dos gestos articulatórios e não à maior velocidade de execução do movimento (Dromey \& Ramig, 1998; Munhall, Ostry \& Parush, 1985; Ostry \& Munhall, 1985), os resultados sugerem que a redução da tensão articulatória ocasionou gestos de menor amplitude.

Um aspecto importante é a relação entre taxa de elocução e hipoarticulação. A hipoarticulação tende a ocorrer em taxas rápidas de elocução (Bradlow et al., 1996; Krause \& Braida, 2002). No inglês americano, por exemplo, a fala claramente articulada apresenta elementos como: ocorrência da fase de soltura das plosivas, VOT longo em palavras iniciadas por plosivas não-vozeadas, amplo espectro vocálico e aumento da energia entre $1 \mathrm{k}$ e $3 \mathrm{k} \mathrm{Hz}$ (Krause \& Braida, 2004). A fala hipoarticulada, por outro lado, tende a suprimir esses elementos acústicos e articulatórios. Neste sentido, o nosso trabalho demonstra que, em PB, pode ocorrer modificações no modo articulatório quando há aumento de taxa de elocução: verificamos cinco ocorrências de plosivas fricativizadas na fala suavizada (todas envolvendo o fone $[\mathrm{p}]$ ). Portanto, o aumento da taxa de elocução favorece a ocorrência de determinados processos articulatórios, os quais caracterizam a fala hipoarticulada.

Pesquisas anteriores também encontraram modificações na duração segmental ao se comparar a fala antes e depois do tratamento com suavização. Em adultos, há achados que indicaram redução na variabilidade da duração das vogais e/ou da taxa de elocução (Onslow, van Doorn \& Newman, 1992; Packman, Onslow \& van Doorn, 1994) e também há achados que indicam aumento na duração média de vogais (Blomgren et al., 1998). Em um grupo de crianças, Riley \& Ingham (2000) encontraram aumento na duração média de vogais, não havendo modificação na duração das plosivas e do VOT. Entretanto, os resultados deste estudo divergem da literatura no sentido de que não foram encontradas diferenças na duração de vogais, apenas na duração de consoantes. Não deixa de ser um resultado surpreendente, porque as vogais tendem a apresentar maior variabilidade duracional em comparação com as consoantes, pelo fato de não apresentarem um ponto específico de articulação (Barbosa, 2006). Os resultados deste trabalho, portanto, sugerem que a estratégia de suavização atua principalmente em 
segmentos consonantais e não em segmentos vocálicos.

\subsection{Frequência Fundamental}

Houve redução de aproximadamente $20 \mathrm{~Hz}$ no F0 no início e no meio das vogais tônicas e também ao longo das palavras-alvo na fala suavizada em comparação com a habitual. Desta forma, a suavização também foi aplicada em nível glótico, ocasionando a redução da tensão muscular nas pregas vocais, mesmo sem ter havido instrução explícita neste sentido. A diminuição do F0 ocorreu muito mais em relação à média do que ao desvio-padrão. Assim, a fala suavizada soou mais grave, mas não mais monótona em comparação com a fala habitual. Tendo em vista que o desvio-padrão, mas não a média, de F0 está correlacionado com a inteligibilidade da fala (Bradlow et al., 1996), conclui-se que a suavização não teve impacto sobre a inteligibilidade da fala ${ }^{4}$ do sujeito.

$\mathrm{Na}$ literatura pesquisada, não foram encontrados artigos que analisassem o efeito da suavização no F0 de sujeitos com gagueira. Entretanto, há achados que indicam redução na média (Bosshardt et al., 1997) e na variabilidade de F0 (Denny \& Smith, 1997) na fala fluente de adultos com gagueira em comparação com adultos sem gagueira.

\subsection{Frequência dos Formantes}

Não houve diferença de F1, F2 e F3 no início e no meio das vogais tônicas quando se comparou a fala habitual com a suavizada. Isso indica que a redução da tensão muscular não alterou a posição dos articuladores durante a produção das sete vogais orais. O espaço vocálico é um fator importante para a inteligibilidade da fala (Bradlow et al., 1996; Krause \& Braida, 2004). F1 realiza a distinção entre vogais altas e baixas, porque seus valores são inversamente proporcionais à altura da língua (Johnson, 1997). F2 realiza a distinção entre vogais anteriores e posteriores, porque seus valores são proporcionais à posição ântero-posterior da língua (Johnson, 1997). F3 está relacionado à ressonância, porque seus valores estão correlacionados com as constrições labial, faríngea e velofaríngea (Johnson, 1997). Portanto, tendo em vista que a suavização não alterou a configuração formântica, conclui-se que não houve mudança na precisão articulatória com o uso da estratégia (Harnsberger et al., 2008).

Prosek et al. (1987) analisaram os primeiros dois formantes vocálicos de adultos com e sem gagueira. Os resultados não indicaram diferenças significativas de F1 e F2 na produção fluente dos adultos com e sem gagueira e na produção disfluente dos adultos com gagueira. Blomgren et al. (1998) analisaram os primeiros dois formantes de vogais emitidas fluentemente por adultos com gagueira que haviam realizado tratamento com suavização, por adultos com gagueira que não haviam realizado tratamento e por adultos sem gagueira. Os grupos não diferiram

\footnotetext{
4. Neste trabalho, a expressão "inteligibilidade de fala" refere-se à facilidade para compreender a fala que foi produzida. Assim, uma fala mais inteligível é mais facilmente compreendida e uma fala menos inteligível é menos facilmente compreendida.
} 
MERLO e BARBOSA - Análise acústica da fala suavizada...

em relação a $F$ 1, mas diferiram em relação a $F$ 2, o qual foi significativamente menor nos sujeitos com gagueira não-tratada. Os resultados desta pesquisa se assemelham aos de Prosek et al. (1987), mas não aos de Blomgren et al. (1998).

\subsection{Inclinação Espectral}

A inclinação espectral foi menor em módulo na fala suavizada em comparação com a fala habitual. Valores menores em módulo estão relacionados ao aumento de energia em altas frequências em relação às baixas frequências. $\mathrm{O}$ maior relaxamento das pregas vocais na fala suavizada (com consequente redução de F0) pode ter aumentado a área de fechamento glotal (Murry, Xu \& Woodson, 1998), ocasionando menor perda de energia e, portanto, menor inclinação espectral (Hanson \& Chuang, 1999). Assim, o grau de soprosidade da fala suavizada do sujeito foi menor em relação à fala habitual (Francis, Ciocca \& Yu, 2003; Hanson \& Chuang, 1999).

A inclinação espectral também está inversamente correlacionada com a inteligibilidade da fala (Krause \& Braida, 2004). Assim, o resultado desta medida também sugere ganhos na inteligibilidade com o uso da suavização.

Não foram encontrados artigos que analisassem o efeito da suavização na inclinação espectral de sujeitos com gagueira. Entretanto, Bohnen \& Recco (2006) encontraram diferenças na configuração laríngea durante a fala de adultos com gagueira não-tratada em comparação com adultos sem gagueira. Dentre os achados, pode-se destacar: a maioria dos sujeitos apresentou espasmos ou tremores laríngeos durante a fala; alguns sujeitos apresentaram fechamento glótico incompleto e dificuldade de coaptação das pregas vocais no início da fonação. Essas diferenças podem gerar perdas de energia, fazendo com que a fala gaguejada apresente maior inclinação espectral em relação à fala fluente.

\section{CONCLUSÕES}

A estratégia de suavização alterou significativamente a prosódia da fala do paciente L.

As plosivas não-vozeadas, as palavras-alvo e as frases apresentaram menor duração na fala suavizada em comparação com a fala habitual. Por outro lado, a fase de soltura das plosivas não-vozeadas e o VOT apresentaram maior duração. Todos esses achados indicam que a fala suavizada foi hipoarticulada em relação à fala habitual.

$\mathrm{O}$ F0 das vogais tônicas e das palavras-alvo foi mais baixo na fala suave em comparação com a habitual. Assim, a suavização também foi aplicada em nível glótico.

Não houve modificação nos valores de F1, F2 e F3 nas vogais orais tônicas das palavras-alvo com o uso da suavização, sugerindo que a estratégia não alterou o posicionamento dos articuladores durante a produção vocálica.

Houve diminuição da inclinação espectral com o uso da suavização, 
Cadernos de EsTUDOS LINGǘlsticos (54.1) - Jan./Jun. 2012

indicando que a estratégia proporcionou aumento de energia em altas frequências.

Portanto, a estratégia de suavização não apenas reduz o número de hesitações gaguejadas presentes na fala, mas também altera significativamente a prosódia da fala. A fala suavizada é mais hipoarticulada, mais grave e com maior concentração de energia em altas frequências em comparação à fala habitual.

\section{REFERÊNCIAS BIBLIOGRÁFICAS}

ALM, P. A. (2004) Stuttering and the basal ganglia circuits: a critical review of possible relations. Journal of Communication Disorders, 37 (4), 325-69. . (2005). On the causal mechanisms of stuttering. PhD thesis. Lund: Lund University.

ANDRADE, C. R. F. de (1999). Tratamento e intervenção precoce no tratamento das gagueiras infantis. Carapicuíba: Pró-Fono.

. (2006). Perfil da fluência da fala: parâmetro comparativo diferenciado por idade para crianças, adolescentes, adultos e idosos. Barueri: Pró-Fono. CD-ROM (Série livros digitais de pesquisas financiadas por agências de fomento).

ARCURI, C. F.; OSBORN, E.; SCHIEFER, A. M. \& CHIARI, B. M. (2009). Taxa de elocução de fala segundo a gravidade da gagueira. Pró-Fono Revista de Atualização Científica, 21 (1), 45-50.

BARBOSA, P. A. (2006). Incursões em torno do ritmo da fala. Campinas e São Paulo: Pontes Editores e Fapesp.

BLOMGREN, M.; ROBB, M. \& CHEN, Y. (1998). A note on vowel centralization in stuttering and nonstuttering individuals. Journal of Speech Language and Hearing Research, 41 (5), 10421051.

BOERSMA, P. \& WEENINK, D. (2009). Praat: doing phonetics by computer (versão 5.1.12) [programa de computador]. Download realizado em 01 de março de 2009, a partir de http://www. praat.org

BOHNEN, A. J. \& RECCO, V. (2006). Características anátomo-fisiológicas do aparelho fonador de pessoas que gaguejam: estudo piloto. In: XIV Congresso Brasileiro de Fonoaudiologia, Salvador. Revista da Sociedade Brasileira de Fonoaudiologia, vol. I.

BOSSHARDT, H. G.; SAPPOK, C.; KNIPSCHILD, M. \& HOLSCHER, C. (1997). Spontaneous imitation of fundamental frequency and speech rate by nonstutterers and stutterers. Journal of Psycholinguistic Research, 26 (4), 425-48.

BOTHE, A. K.; DAVIDOW, J. H.; BRAMLETT, R. E. \& INGHAM, R. J. (2006). Stuttering treatment research 1970-2005: I. systematic review incorporating trial quality assessment of behavioral, cognitive, and related approaches. American Journal of Speech-Language Pathology, 15, 321341 .

BRADLOW, A. R.; TORRETTA, G. M. \& PISONI, D. B. (1996). Intelligibility of normal speech I: global and fine-grained acoustic-phonetic talker characteristics. Speech Communication, 20, 255-272.

COLACICCO, F. B.; SCHIEFER, A. M. \& OSBORN, E. (2005). Medidas acústicas de duração da fala de indivíduos gagos e não gagos. Fono Atual, 8(31), 26-30.

DENNY, M. \& SMITH, A. (1997). Respiratory and laryngeal control in stuttering. In: Curlee, R. F. 
MERLO e BARBOSA - Análise acústica da fala suavizada...

\& Siegel, G. M. (eds). Nature and treatment of stuttering: new directions. 2nd ed. Boston: Allyn and Bacon. 128-142.

DROMEY, C. \& RAMIG, L. O. (1998). Intentional changes in sound pressure level and rate: their impact on measures of respiration, phonation, and articulation. Journal of Speech Language and Hearing Research, 41 (5), 1003-18.

FARNETANI, E. (1997). Coarticulation and connected speech processes. In: Hardcastle, W. J. \& Laver, J. (eds). The handbook of phonetic sciences. Oxford: Blackwell Publishers. 371-404.

FRANCIS, A. L.; CIOCCA, V. \& YU, J. M. C. (2003). Accuracy and variability of acoustic measures of voicing onset. Journal of the Acoustical Society of America, 113 (2), 1025-1032.

HANSON, H. M. \& CHUANG, E. S. (1999). Glottal characteristics of male speaker: acoustic correlates and comparison with female data. Journal of the Acoustical Society of America, 106 (2), 1064-1077.

HARNSBERGER, J. D.; WRIGHT, R. \& PISONI, D. B. (2008). A new method for eliciting three speaking styles in the laboratory. Speech Communication, 50, 323-336.

IMBRIE, A. K. K. (2005). Acoustical study of the development of stop consonants in children. PhD thesis. Massachusetts Institute of Technology.

JOHNSON, K. (1997). Acoustic \& auditory phonetics. Cambridge (MA) e Oxford: Blackwell Publishers.

KRAUSE, J. C. \& BRAIDA, L. D. (2002). Investigating alternative forms of clear speech: the effects of speaking rate and speaking mode on intelligibility. Journal of the Acoustical Society of America, 112 (5), 2165-2172.

KRAUSE, J. C. \& BRAIDA, L. D. (2004). Acoustic properties of naturally produced clear speech at normal speaking rates. Journal of the Acoustical Society of America, 115 (1), 362-378.

MARCHESAN, I. Q. (1998). Fundamentos em Fonoaudiologia - aspectos clínicos da motricidade oral. Rio de Janeiro: Guanabara Koogan.

McCREA, C. R. \& MORRIS, R. J. (2005). The effects of fundamental frequency level on voice onset time in normal adult male speakers. Journal of Speech, Language, and Hearing Research, 48 (5), 1013-1024.

MEIRA, I. (1997). Gagueira. In: Goldfeld, M. Fundamentos em Fonoaudiologia: linguagem. Rio de Janeiro: Guanabara Koogan. p. 53-68.

MUNHALL, K. G.; OSTRY, D. J. \& PARUSH, A. (1985). Characteristics of velocity profiles of speech movements. Journal of Experimental Psychology Hum Percept Perform, 11(4), 457-74.

MURRY, T.; XU, J. J. \& WOODSON, G. E. (1998). Glottal configuration assoaciated with fundamental frequency and vocal register. Journal of Voice, 12 (1), 44-49.

OLIVEIRA, C. M. C. de. (2007). Gagueira familial: repercussões clínicas. In: Rocha, E. M. N. (Coord.). Gagueira: um distúrbio de fluência. São Paulo: Editora Santos. 107-131.

ONSLOW, M.; VAN DOORN, J. \& NEWMAN, D. (1992). Variability of acoustic segment durations after prolonged-speech treatment for stuttering. Journal of Speech and Hearing Research, 35 (3), 529-36.

PACKMAN, A.; ONSLOW, M. \& VAN DOORN, J. (1994). Prolonged speech and modification of stuttering: perceptual, acoustic, and electroglottographic data. Journal of Speech and Hearing 
Research, 37 (4), 724-37.

PROSEK, R. A.; MONTGOMERY, A. A.; WALDEN, B. E. \& HAWKINS, D. B. (1987). Formant frequencies of stuttered and fluent vowels. Journal of Speech and Hearing Research, 30 (3), 301-305.

RILEY, G. D. \& INGHAM, J. C. (2000). Acoustic duration changes associated with two types of treatment for children who stutter. Journal of Speech, Language, and Hearing Research, 43(4), $965-78$.

ST. LOUIS, K. O. \& MYERS, F. L. (1997). Management of cluttering and related fluency disorders. In: Curlee, R. F. \& Siegel, G. M. (eds). Nature and treatment of stuttering: new directions. 2nd ed. Boston: Allyn and Bacon. 313-332.

OSTRY, D. J. \& MUNHALL, K. G. (1985). Control of rate and duration of speech movements. Journal of Acoustical Society of América, 77(2), 640-8.

PEREIRA, L. D. (1993). Processamento Auditivo. Temas sobre Desenvolvimento, 11, 7-14.

PRINS, D. (1997). Modifying stuttering - the stutterer's reactive behavior: perspectives on past, present, and future. In: Curlee, R. F. \& Siegel, G. M. (eds). Nature and treatment of stuttering: new directions. 2nd ed. Boston: Allyn and Bacon. 335-355.

RAMIG, P. R. \& BENNETT, E. M. (1997). Clinical management of children: direct management strategies. In: Curlee, R. F. \& Siegel, G. M. (Eds). Nature and treatment of stuttering: new directions. $2^{\text {nd }}$ ed. Boston: Allyn and Bacon. 292-312.

RILEY, G. D. \& INGHAM, J. C. (2000). Acoustic duration changes associated with two types of treatment for children who stutter. Journal of Speech, Language and Hearing Research, 43(4), 965-78.

YARUSS, S. (2004). Speech disfluency and stuttering in children. In: Kent, R. D. (Ed.). The MIT encyclopedia of communication disorders. Cambridge (MA) e Londres: A Bradford Book e The MIT Press. 180-183. 
MERLO e BARBOSA - Análise acústica da fala suavizada...

Ficha técnica

$\begin{aligned} \text { Divulgação } & \text { Publicações IEL-UNICAMP } \\ \text { Montagem } & \text { Publicações IEL } \\ \text { Editoração } & \text { In Design CS5 } \\ \text { Formato } & 16 \times 23 \mathrm{~cm} \\ \text { Mancha } & 12 \times 19 \mathrm{~cm} \\ \text { Tipologia } & \text { Time new roman } \\ \text { Papel } & \text { Miolo: pólen soft } 75 \mathrm{~g} / \mathrm{m} \\ & \text { Capa: cartão supremo } 250 \mathrm{~g} / \mathrm{m} \\ \text { Impressão e acabamento } & \text { Oficinas Gráficas da UNICAMP } \\ \text { Numero de páginas } & 182 \text { páginas } \\ \text { Tiragem } & 300 \text { exemplares }\end{aligned}$

